\title{
Effects of microRNA-125b on multiple myeloma cell growth in vitro and in vivo
}

\author{
YANXIA JIANG ${ }^{1}$, JIANGHUA DING ${ }^{2}$, JING LI $^{1}$ and GUOAN CHEN ${ }^{1}$ \\ ${ }^{1}$ Hematology Department, The First Affiliated Hospital of Nanchang University, Nanchang, Jiangxi 330000; \\ ${ }^{2}$ Hematology Department, The 171st Affiliated Hospital of Jiujiang University, Jiujiang, Jiangxi 332000, P.R. China
}

Received February 12, 2018; Accepted August 17, 2018

DOI: $10.3892 / o r .2018 .6668$

\begin{abstract}
Multiple myeloma (MM) is a heterogeneous disease with a poor prognosis. The expression of microRNA-125b (miR-125b), a novel oncomiR, is elevated in various cancer types. The present study found that the expression of miR-125b was increased in plasma samples from 35 patients with MM, compared with that in samples from 20 healthy controls, by performing real-time PCR. CCK- 8 assay, migration and invasion assay showed that the downregulation of miR-125b inhibited cell proliferation and migration and reduced the levels of phosphorylated Akt, compared with those of the blank and negative control groups. Dual-Luciferase activity assay demonstrated that the tumor suppressor $\mathrm{PH}$ domain and leucine rich repeat protein phosphatase 2 (PHLPP2) was a target of miR-125b, which inhibited PHLPP2 and directly bound to the 3'untranslated region of PHLPP2, resulting in elevated Akt signaling. Furthermore, the expression of a miR-125b inhibitor in MM cells in a xenograft mouse model suppressed tumor growth. These results showed that the inhibition of miR-125b suppressed MM progression by inhibiting Akt signaling, and suggested that miR-125b may be a novel molecular therapeutic target for MM treatment.
\end{abstract}

\section{Introduction}

Multiple myeloma (MM) is a clonal B-cell malignancy characterized by the proliferation of plasma cells (PCs) within the bone marrow (BM). MM is the second most common hematological malignancy, but it remains incurable, with a survival rate ranging between a few months and over 10 years $(1,2)$. Although treatment strategies have evolved from traditional chemotherapy and autologous hematopoietic stem cell transplantation to novel targeted drug therapies, patient outcomes

Correspondence to: Professor Guoan Chen, Hematology Department, The First Affiliated Hospital of Nanchang University, 17 Yongwaizheng Street, Nanchang, Jiangxi 330000, P.R. China E-mail: guoanchen010@sina.com

Key words: miroRNA-125b, multiple myeloma, metastasis, cell proliferation, cell apoptosis, cell migration have not improved significantly (3). The 5-year survival rate remains at only $30-40 \%$, mainly due to the development of drug resistance (4). Therefore, identifying the mechanisms that regulate the key genes responsible for the progression of $\mathrm{MM}$ and the underlying mechanisms governing the malignant behavior of MM cells is important for establishing treatments to improve patient prognosis. One topic of interest is chromosomal abnormalities and other types of genetic or epigenetic alterations that may contribute to microRNA (miRNA) dysregulation in cancer (5-7).

miRNAs, a newly identified class of endogenous, small, non-coding RNAs, regulate gene expression by inhibiting protein translation or by inducing mRNA degradation (5). miRNAs are involved in various biological processes, including cellular growth, development, metabolism, apoptosis and development (8). Increasing evidence has demonstrated that differential miRNA expression, including that of miRNA (miR)-21, miR-155, miR-17-92 and miR-125a-5p, is involved in $\mathrm{MM}$, suggesting the importance of miRNAs in the progression of MM (9-11). Deregulated miRNAs can act as oncogenes or tumor suppressors, with the former being upregulated and the latter being downregulated in cancer cells (12-14). miR-125b is an important member of the miRNA family, which is involved in various types of human, including prostate (15), colorectal cancer (16), leukemia (17), breast (18) and lung cancer (19). However, the biological role of miR-125b in MM remains to be elucidated. Several studies have suggested that the dysregulation of miRNAs initiates activation of the Akt signaling pathway, which is involved in the progression of breast, bladder and non-small cell lung cancer (20-23). However, the biological role of miR-125b and its effects on the Akt signaling pathway in the pathogenesis and progression of MM remain to be fully elucidated. The aim of the present study was to characterize the expression of miR-125b in MM and identify its function in MM cell lines in vitro and in vivo, and to determine the effect of miR-125b on the Akt signaling pathway. Finally, screening for target genes of miR-125b was performed. It was found that miR-125b promoted MM cell proliferation by targeting tumor suppressor $\mathrm{PH}$ domain and leucine rich repeat protein phosphatase 2 (PHLPP2) and that the downregulation of PHLPP2 rescued the effect of an miR-125b inhibitor in MM cells. These findings provide novel insights into the function of miR-125b during the development of MM and suggest that this miRNA is involved in tumorigenesis. 


\section{Materials and methods}

Study subjects and sample collection. The specimens used in the present study were obtained from 41 patients who were diagnosed according to the National Comprehensive Cancer Network clinical practice guidelines for MM at the First Affiliated Hospital of Nanchang University (Nanchang, China) between October 2015 and July 2017 (24). Plasma samples from 41 patients diagnosed with MM were assessed; six samples were used for microarray tests, and 35 samples formed the validation set. Any patients who received chemotherapy and/or biotherapy were excluded from the study, and those with other types of malignant tumor were also eliminated. To investigate the expression of miR-125b in different genetic subtypes of MM, 35 samples were cytogenetically classified using the FISH technique (Abbott Diagnostics, Berkshire, UK). Cytogenetic abnormalities involving $13 q$ deletions and immunoglobulin heavy-chain gene rearrangements were investigated. The patient details are shown in Tables I and II. In addition, 20 plasma samples in the validation set from healthy individuals were used as a control. Venous blood was collected in EDTA tubes (BD Biosciences, Franklin Lakes, NJ, USA). The blood samples were centrifuged at $800 \mathrm{x}$ g for $10 \mathrm{~min}$. The plasma was then transferred into RNase/DNase-free tubes, followed by a 10-min high-speed centrifugation at $16,000 \mathrm{x} \mathrm{g}$ to completely remove the cell debris. The plasma was transferred to a fresh tube and stored at $-80^{\circ} \mathrm{C}$ on being frozen with liquid nitrogen. The present study was approved by the Medical Research Ethics Committee of The First Affiliated Hospital of Nanchang University, and written informed consent was obtained from all the study subjects. The IRB number of human sample study approved by the institutional committee is 2015(096).

miRNA array. Total RNA was extracted from six patients with MM and six healthy controls using the phenol-chloroform method (TRIzol; Invitrogen; Thermo Fisher Scientific, Inc., Waltham, MA USA). The quality of the RNA was assessed by capillary electrophoresis (Agilent Technologies Inc., Santa Clara, CA, USA). Libraries for small RNA sequencing were prepared using the NEBNext Multiplex Small RNA Library Prep Set for Illumina (New England BioLabs, Inc., Ipswich, MA, USA) according to the manufacturer's protocol. The libraries were quantified using the Agilent Bioanalyzer 2100 system with DNA high-sensitivity chips. The raw sequence files were subjected to quality control analysis with the Fast QC quality control tool. To avoid low-quality data, adaptors were removed by Cutadapt (version 1.2.1; DOI:10.14806/ej.17.1.200), and lower-quality sequences were trimmed. The clean reads were screened at a length of 21-22 nt as miRNA and were located to the reference sequence with Bowtie software (version 2; CGE Risk Management Solutions B.V., Leidschendam, The Netherlands). The functions of novel miRNAs were analyzed with miRDeep2 software (version 2.0.0.8; https:www. mdc-berlin.de/content/mirdeep2-documentation). The differential expression sequence was used to calculate differential expression levels and to evaluate the statistical significance of detected alterations between the control and case samples.
Table I. Summary of the clinical details of 35 patients with multiple myeloma used for reverse transcription-quantitative polymerase chain reaction analysis.

\begin{tabular}{lc}
\hline Clinical details & $\mathrm{n}(\%)$ \\
\hline Sex & \\
Male & $23(65.7)$ \\
Female & $12(34.3)$ \\
Age range (years) & $35-75$ \\
Mean age (years) & 59 \\
Durie-Salmon stage & \\
I & $10(28.57)$ \\
II & $6(17.14)$ \\
III & $19(54.29)$ \\
Karyotype & \\
t (4:14) & $9(25.71)$ \\
$t(11: 14)$ & $5(14.28)$ \\
$t(14: 16)$ & $6(17.14)$ \\
del (13q) as a unique abnormality & $10(28.57)$ \\
Normal FISH & $5(14.29)$ \\
\hline
\end{tabular}

RNA isolation from human plasma and reverse transcriptionquantitative polymerase chain reaction ( $R T-q P C R)$ analysis. Total RNA was extracted from human plasma using the mirVana PARIS RNA Isolation kit (Ambion; Thermo Fisher Scientific, Inc.) following the manufacture's protocol for liquid samples. The concentration and purity of the extracted RNA were measured at 260 and $280 \mathrm{~nm}$ optical densities (ODs). cDNA was synthesized from the RNA for RT-qPCR analysis using gene-specific primers (Applied Biosystems; Thermo Fisher Scientific, Inc.) with the M-MLV Reverse Transcriptase kit (GeneCopoeia, Inc., Rockville, MD, USA) according to the manufacturer's protocol. To determine the expression levels of miR-125b, qPCR analysis was performed with SYBR Green (Takara Bio, Inc., Osaka, Japan). $\beta$-actin and U6 were used as internal controls. The PCR conditions were as follows: 40 cycles of $95^{\circ} \mathrm{C}$ for $5 \mathrm{~min}, 95^{\circ} \mathrm{C}$ for $45 \mathrm{sec}$, $55^{\circ} \mathrm{C}$ for $15 \mathrm{sec}$, and $72^{\circ} \mathrm{C}$ for $50 \mathrm{sec}$, in which each reaction $(25 \mu \mathrm{l})$ contained cDNA $(100 \mathrm{ng})$, primers $(10 \mu \mathrm{M})$ and $10 \mathrm{X}$ Ex Taq E buffer $(25 \mu \mathrm{l})$. The samples were analyzed in triplicate, and gene expression was quantified by normalizing target gene expression to that of the internal control using the $2^{-\Delta \Delta C q}$ formula (25). The primer sequences used were as follows: miR-125b, forward 5'-TGCGCTAAAGTGCTTATAGTG C-3' and reverse 5'-CCAGTGCAGGGTCCGAGGTATT-3'; PHLPP2, forward 5'-CCAATGAGCAAGGACAGGAT-3' and reverse 5'-GGTCCTCTGGTTCCATCTGA-3'; $\beta$-actin, forward 5'-AGCGAGCATCCCCCAAAGTT-3' and reverse 5'-GGGCACGAAGGCTCATCATT-3'; and U6, forward 5'-CTCGCTTCGGCAGCACA-3' and reverse 5'-AACGCT TCACGAATTTGCG-3'. The samples were analyzed in triplicate, and gene expression was quantified by normalizing target gene expression to that of the internal control using the $2^{-\Delta \Delta C q}$ formula. 
Table II. Association between the expression of miR-125b and the clinical pathological characteristics of patients with multiple myeloma.

\begin{tabular}{lccc}
\hline Characteristic & Cases $(\%)$ & miR-125b expression & P-value \\
\hline Age (years) & & & 0.241 \\
$\leq 50$ & 16 & $1.66 \pm 0.03$ & \\
$>50$ & 19 & $1.72 \pm 0.05$ & 0.439 \\
Sex & & & $1.68 \pm 0.03$ \\
$\quad$ Male & 23 & $1.72 \pm 0.05$ & $\mathbf{0 . 0 0 9}$ \\
Female & 12 & & \\
Durie-Salmon stage & & $1.62 \pm 0.04$ & $\mathbf{0 . 0 1 2}$ \\
I/II phase & 16 & $1.76 \pm 0.03$ & \\
III phase & 19 & $1.82 \pm 0.05$ & 0.455 \\
Extramedullary infiltration & & $1.66 \pm 0.03$ & \\
$\quad$ Yes & 8 & $1.65 \pm 0.12$ & $1.72 \pm 0.03$ \\
$\quad$ No & 27 & & \\
Karyotype & 5 & &
\end{tabular}

$\mathrm{P}$-values in bold are statistically significant $(\mathrm{P}<0.05)$. miR, microRNA.

Cell lines and transfection. Human MM cell lines (MM.1S, U266, and RPMI-8226) and normal plasma cells (nPCs) were cultured in RPMI-1640 (Gibco; Thermo Fisher Scientific, Inc.) supplemented with $10 \%$ fetal bovine serum (FBS; HyClone Laboratories, Logan, UT, USA) and $1 \%$ penicillin/streptomycin at $37^{\circ} \mathrm{C}$ in a $5 \% \mathrm{CO}_{2}$ atmosphere. When the cells reached $80 \%$ confluency, cells at the logarithmic growth phase were collected. The cell lines were obtained from American Type Culture Collection (Manassas, VA, USA). The cells were assigned to the following groups: Blank group (no transfection), negative control (NC) group (transfected with the miR-125b NC sequence), miR-125b mimic (transfected with miR-125b mimic), and miR-125b inhibitor (transfected with miR-125b inhibitor). The cells were also transfected with small interfering (si)RNA against PHLPP2 (siPHLPP2) (5'-CCGGAATTCCAATCCCTAAATTTCCCT GGGA-3'). All sequences were purchased from Shanghai GenePharma Co, Ltd. (Shanghai, China). Transfection of plasmids was performed using Lipofectamine 2000 reagent (Invitrogen; Thermo Fisher Scientific, Inc.) according to the manufacturer's protocol.

Cell proliferation analysis. For the cell proliferation assays, the cells were plated in individual wells of a 96-well plate (1,500 cells/well) and were examined $48 \mathrm{~h}$ following transfection using a Cell Counting Kit-8 (CCK-8; Dojindo Molecular Technologies, Inc., Shanghai, China). The OD values were determined at a wavelength of $450 \mathrm{~nm}$ using a microplate reader. For the survival rate assay, the number of viable cells was counted using Trypan blue dye (Beijing Solarbio Science $\&$ Technology Co. Ltd., Beijing, China) and a Countstar Cell Counter (Inno-Alliance Biotech, Inc. Wilmington, DE, USA) according to the manufacturer's protocol. All assays were performed in triplicate.

Migration and invasion assays. For the migration experiment at $72 \mathrm{~h}$ post-transfection, the cells were collected and resuspended in RPMI-1640 medium (Gibco; Thermo Fisher Scientific, Inc.), following which they were inoculated into the Transwell upper chamber and placed in an incubator at $37^{\circ} \mathrm{C}$ with $5 \% \mathrm{CO}_{2}$ for $48 \mathrm{~h}$. The cells that failed to penetrate the upper chamber were removed, and the membrane was fixed in $95 \%$ ethanol for $15-20 \mathrm{~min}$ and then immersed in water. The membrane was stained with crystal violet for $10 \mathrm{~min}$ and re-immersed in water, followed by observation and image capture under a high-magnification laser confocal microscope (ZEISS LSM888; Carl Zeiss, Jena, Germany). The number of cells on the back of the membrane was then calculated. Five high-power fields were randomly selected, and the number of cells penetrating through the polycarbonate membrane was used to evaluate the cell migration ability. For the invasion experiment, Matrigel matrix was dissolved at $4^{\circ} \mathrm{C}$ overnight and diluted 1:3 with serum-free DMEM. A total volume of $30 \mu \mathrm{l}$ of Matrigel was added to the Transwell upper chamber until it covered the bottom of the upper chamber. The cell suspension was added to the upper chamber, and $0.5 \mathrm{ml}$ of DMEM (Beijing Solarbio Science \& Technology Co., Ltd., Beijing, China) containing 10\% FBS was added to the lower chamber of the 24-well plate. The number of cells penetrating through the Matrigel was determined to assess the cell invasion ability.

Apoptosis analysis. The cells in each group were collected at 24,48 and $72 \mathrm{~h}$ post-transfection, and cold phosphate-buffered 
saline (PBS) was used to wash the cells three times. The cells were resuspended with $500 \mu \mathrm{l}$ of precooled binding buffer at a concentration of $5 \times 10^{6}$ cells $/ \mathrm{ml}$. The cell suspension $(100 \mu \mathrm{l})$ was added to flow cytometry tubes, and $5 \mu \mathrm{l}$ of Annexin V-fluorescein isothiocyanate (Beyotime Institute of Biotechnology, Haimen, China) was added. Following mixing, the samples were incubated at room temperature in the dark for $15 \mathrm{~min}$, and $5 \mathrm{~min}$ prior to measurements, $5 \mu \mathrm{l}$ of $10 \mathrm{mg} / \mathrm{l}$ propidium iodide (PI) dye (Beyotime Institute of Biotechnology) was added. The samples were immediately analyzed with a FACSort system without washing or fixation. Each assay was repeated three times.

Luciferase reporter analysis. The pGL3-PHLPP2-3' untranslated region (3'UTR)-WT/MUT vector (Guangzhou Ribobio, Co., Ltd., Guangzhou, China) was cotransfected with the control plasmid or miR-125b-expressing plasmid into 293T cells (Guangzhou Ribobio, Co., Ltd.) using Lipofectamine 2000 (Thermo Fisher Scientific, Inc.). Firefly and Renilla luciferase activities were measured consecutively $24 \mathrm{~h}$ following transfection using the Dual-Luciferase assay kit.

Western blot analysis. Whole-cell protein extracts were obtained from the MM cell lines. The cell lysates were loaded and separated via polyacrylamide gel electrophoresis. The proteins were transferred onto membranes using a Trans-Blot Turbo Transfer Starter system (Bio-Rad Laboratories, Inc., Hercules, CA, USA) for $7 \mathrm{~min}$. Following protein transfer, the membranes were probed with primary antibodies. Cells in the logarithmic growth phase at $80 \%$ confluency were collected, and radioimmunoprecipitation assay lysis buffer containing protease inhibitors (Beyotime Institute of Biotechnology) was used for conventional extraction of total cellular protein. A bicinchoninic acid protein assay kit (Beyotime Institute of Biotechnology) was used for protein quantification. Total proteins $(100 \mu \mathrm{g})$ were separated by $10 \%$ SDS gel electrophoresis under denaturing and non-reducing conditions and then were transferred onto a nitrocellulose membrane. Following the addition of $5 \%$ skim milk, the membranes were agitated in a sealed container for $1 \mathrm{~h}$. The blotted membranes were incubated with the following antibodies: Anti-Akt (1:500; cat. no. sc-5298), anti-PHLPP2 (1:300; cat. no. sc-71973) and anti-phosphorylated (p)-Akt (1:500; cat. no. sc-7985-R), all of which were obtained from Santa Cruz Biotechnology, Inc. (Dallas, TX, USA), and then probed with a goat anti-rabbit secondary antibody (1:3,000; cat. no. sc-2030; Santa Cruz Biotechnology, Santa Cruz, CA, USA), $\beta$-actin (1:200; cat. no. sc-58673; Abcam, Cambridge, MA, USA) was used as a loading control. The immunoblots were visualized using the ImageQuant LAS 4000 digital imaging system (GE Healthcare, Chicago, IL, USA).

Tumor xenograft model and immunohistochemistry. Briefly, $5 \times 10^{6}$ U266 cells were subcutaneously injected into the left upper flank region of male nude mice BALB/c (3-4 weeks of age). Male BALA/C nude mice $(n=20)$ were purchased from the Beijing Hua Fukang Bioscience Company (Beijing, China) and housed under specific pathogen-free conditions. The housing condition were the following: Light-dark cycle was setting as $10 \mathrm{~h}$ of light and $14 \mathrm{~h}$ of dark every day; temperature was maintained at $26-28^{\circ} \mathrm{C}\left(78-82^{\circ} \mathrm{F}\right)$; relative humidity was 40-60\%. Tumor growth was monitored every 5 days. At 6 weeks post-implantation, the mice were sacrificed by cervical dislocation, and tumor size was calculated as follows: Tumor volume $\left(\mathrm{mm}^{3}\right)=\left(\mathrm{Lx} \mathrm{W}^{2}\right) / 2$, where $\mathrm{L}$ is the long axis, and $\mathrm{W}$ is the short axis. All animal experiments were undertaken in accordance with the National Institute of Health Guide for the care and use of laboratory animals, with the approval of the Affiliated Hospital of Nanchang University, the number of the animal experiment approved by the institutional committee is 2017(095). The tissues were cut into $4-\mu \mathrm{m}$ sections by serial sectioning, and the sections were dewaxed, alcohol rehydrated and subjected to microwave antigen retrieval. The tissue samples were immersed in $3 \%$ hydrogen peroxide to block endogenous peroxidase activity. The expression levels of Akt, PHLPP2 and Ki-67 in the local tumor issues were determined by immunostaining with antibodies against Akt (1;200; cat. no. sc-5298), PHLPP2 (1:100; cat. no. sc-71973) and Ki-67 (1:100) (rabbit monoclonal antibody, cat. no. ab16667; Abcam). After incubation for $20 \mathrm{~min}$ at room temperature with the addition of polymerase auxiliary agent, horseradish peroxidase-labeled goat anti-mouse or goat anti-rabbit secondary antibody (cat. nos. ab6789 and ab6721; Abcam, Inc., Cambridge, MA, USA) was added, and the sections were incubated at room temperature for $30 \mathrm{~min}$ and stained with diaminobenzidine (DAB) from the Sigma-Aldrich; EMD Millipore, Billerica, MD, USA). The sections were counterstained with hematoxylin and then mounted. PBS was used instead of a primary antibody in the NC, and normal mucosa was used as a positive control. Cytoplasm and cell membranes containing brown-yellow granules were defined as having positive expression of Akt, PHLPP2 and Ki-67 proteins, and nuclei containing brown-yellow or brown staining indicated positive expression of Akt, PHLPP2 and Ki-67 proteins. The integral optical density (IOD) level of the protein of interest was quantified using Gel-Pro-Analyzer software 6.0 (Media Cybernetics, Inc., Rockville MD, USA).

Statistical analysis. Each experiment was performed at least three times, and the values are reported as the mean \pm standard deviation. Differences between two groups were evaluated using the unpaired t-test. For multiple-group comparisons, one-way analysis of variance was followed by Tukey's multiple comparisons test. $\mathrm{P}<0.05$ was considered to indicate a statistically significant difference. Event-free survival (EFS) curves were plotted separately for patients with low, vs. high expression of miR-125b using the Kaplan-Meier method. The curves were compared by log-rank analysis (GraphPad Prism version 5.0; GraphPad Software, Inc., La Jolla, CA, USA). The EFS times were calculated from the time of diagnosis to the date of relapse, mortality, or last contact. $\mathrm{P}<0.05$ was considered to indicate a statistically significant difference.

\section{Results}

miR-125b is overexpressed in plasma from MM patients and in MM cell lines. The miRNA microarray analysis revealed that miR-125b was significantly upregulated in MM plasma (Fig. 1A). To confirm this result, RT-qPCR analysis was performed in 35 plasma samples from patients with MM 


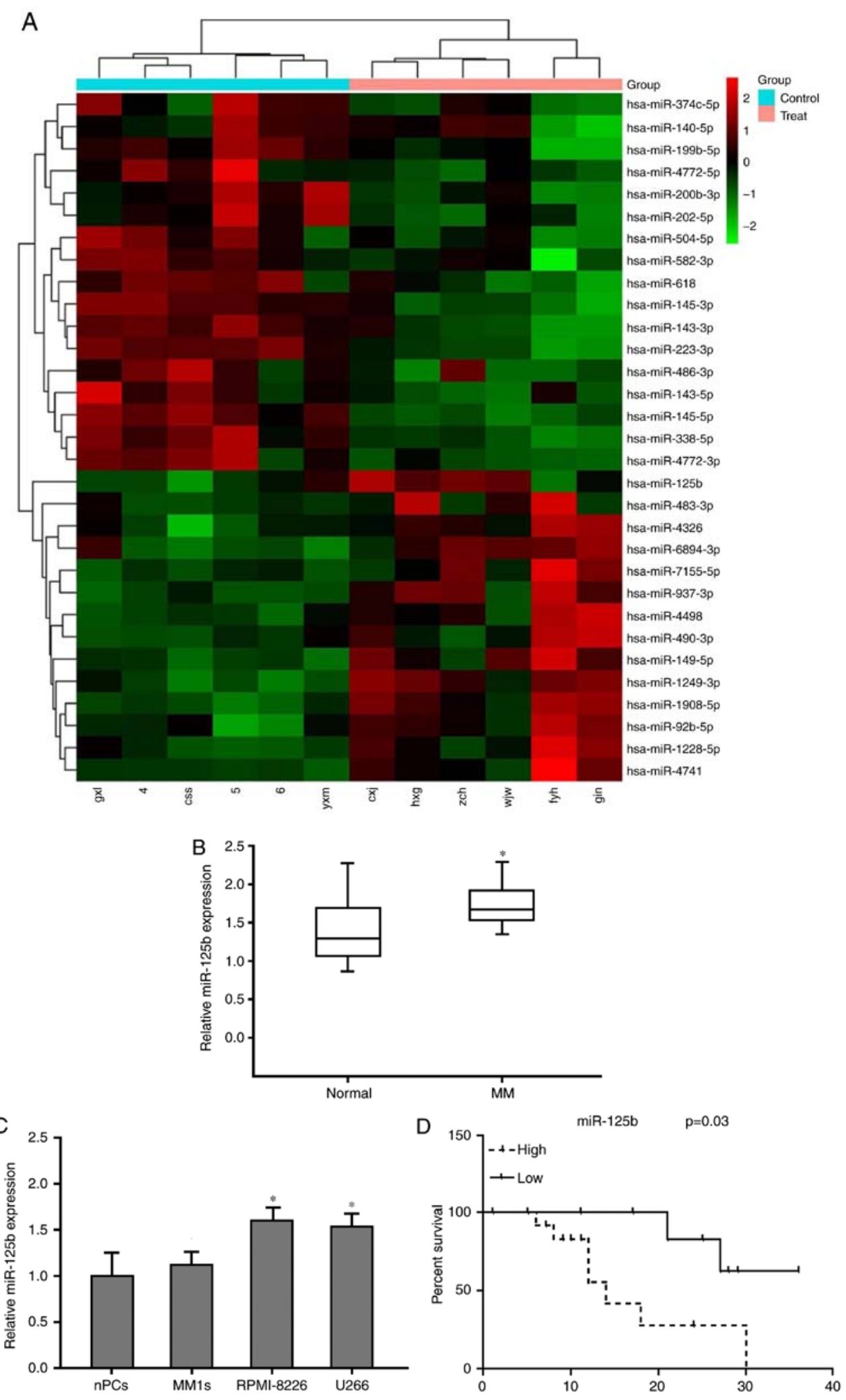

Figure 1. Determination of the expression of miR-125b in human MM cell lines and clinical samples via RT-qPCR analysis. (A) miRNA microarray of deregulated miRNAs in plasma from patients with MM. (B) RT-qPCR detection of miR-125b in PCs derived from the bone marrow of healthy donors (normal) and patients with MM. (C) Expression of miR-125b in MM.1S, U266 and RPMI-8226 MM cell lines, compared with that in nPCs. Each sample was analyzed in triplicate, and error bars represent the standard deviation. ${ }^{*} \mathrm{P}<0.05$. (D) Kaplan-Meier survival curves of event-free survival in patients with MM based on high or low (median) miRNA expression levels. m, months; miR, microRNA; MM, multiple myeloma; RT-qPCR, reverse transcription-quantitative polymerase chain reaction; nPCs, normal plasma cells.

and 20 samples from healthy control individuals. It was found that the level of circulating miR-125b was upregulated in cells from the MM group, compared with those from the control subjects. The normalized expression levels of miR-125b in 



Figure 2. Regulatory effects of miR-125b inhibitor on MM cell proliferation, migration, invasion and apoptosis. (A) Cell Counting Kit-8 assay showed that RPMI-8226 cells and U266 cells transfected with miR-125b inhibitor grew more slowly than NC-transfected and blank cells. (B) Transwell migration assays of RPMI-8226 cells and U266 cells were performed following transfection with miR-125b inhibitor, NC and blank. Cells transfected with miR-125b inhibitor exhibited significantly lower motility than control-treated and blank cells. (C) Invasion ability of RPMI-8226 cells and U266 cells following transfection in each group. (D) Cell apoptosis analysis. (E) Apoptotic rates of transfected cells in each group. NC were used as controls. Data are presented as the mean \pm standard deviation $(\mathrm{n}=3) .{ }^{* *} \mathrm{P}<0.01,{ }^{*} \mathrm{P}<0.05$ vs. the $\mathrm{NC}$ group. miR, microRNA; MM, multiple myeloma; $\mathrm{NC}$, negative control.

the patients with $\mathrm{MM}$ and the control subjects were $1.69 \pm 0.03$ and $1.30 \pm 0.08$, respectively, and the expression of miR-125b in the patients with $\mathrm{MM}$ was significantly higher than that in the control individuals $(\mathrm{P}<0.05)($ Fig. $1 \mathrm{~B})$. Consistent with 
A
PHLPP2-3' UTR WT 5'CCAUUCCCGUGGUGUUCAGGGAU-3' IIIIII| has-miR-125b $\quad 3^{\prime}$ AGUGUUCAAUCCCAGAGUCCCU $-5^{\prime}$ PHLPP2-3' UTR MUT 5' CCAUUCCCGUGGUGAGTCCCAU $-3^{\prime}$

C

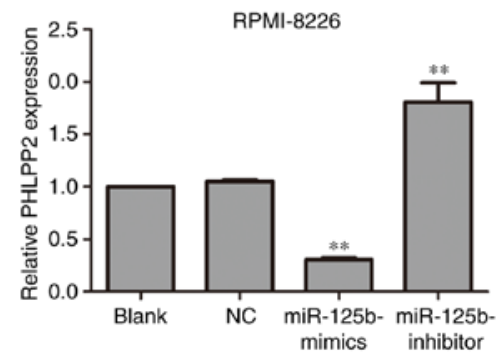

E


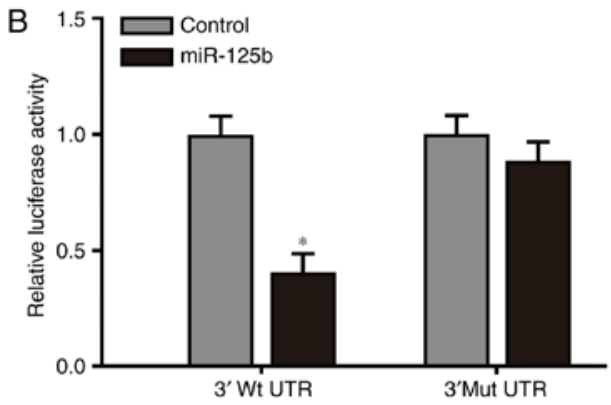

D
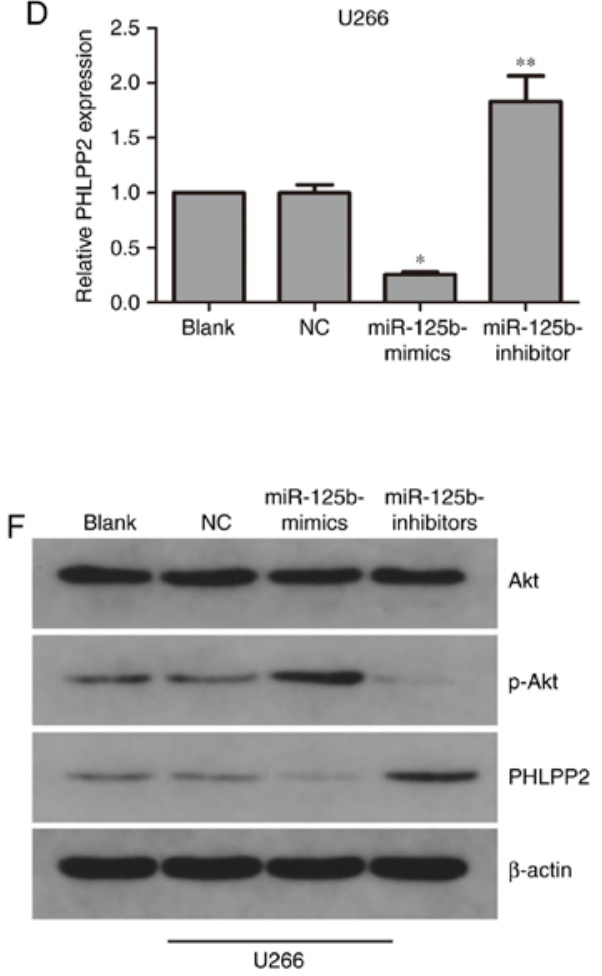

Figure 3. miR-125b suppresses PHLPP2 and augments the AKT pathway. (A) Schematic of miR-125b bound to the PHLPP2 3'-UTR. (B) Relative luciferase activity detected by the dual-luciferase reporter gene activity assay. (C) mRNA levels of PHLPP2 were detected by RT-qPCR analysis in RPMI-8226 cells transfected with miR-125b inhibitor or miR-125b mimic. (D) mRNA levels of PHLPP2 were detected by RT-qPCR analysis in U266 cells transfected with miR-125b inhibitor or miR-125b mimic. (E) Histogram of protein expression levels in each group of U266 cells. (F) Protein expression levels of PHLPP2, Akt and p-Akt were detected by western blot analysis. Diagram of the protein bands in each group detected by western blot analysis of U266 cells compared with the blank and NC groups. NC were used as controls. ${ }^{* *} \mathrm{P}<0.01,{ }^{*} \mathrm{P}<0.05$ vs. the NC group. miR, microRNA; MM, multiple myeloma; UTR, untranslated region; PHLPP2, PH domain and leucine rich repeat protein phosphatase 2; p-Akt, phosphorylated Akt; RT-qPCR, reverse transcription-quantitative polymerase chain reaction; $\mathrm{NC}$, negative control.

these data, the expression of miR-125b in the U266, MM.1S, and RPMI-8226 MM cell lines was significantly increased compared with the expression in the normal, healthy bone marrow-derived PCs (Fig. 1C). The expression levels of circulating miR-125b were significantly higher in patients with MM with extramedullary infiltration $(1.82 \pm 0.05)$ than in patients without extramedullary infiltration $(1.66 \pm 0.03)$, and the expression level in stage III patients $(1.76 \pm 0.03)$ was significantly higher than that in stage I/II patients $(1.62 \pm 0.04)$ (both $\mathrm{P}<0.05)$. However, there was no association between the expression of miR-125b with and the age, sex or karyotype of the patients (all $\mathrm{P}>0.05$; Table II).

Association between the expression of miR-125b and EFS. Kaplan-Meier analyses were performed to assess the association between the expression levels of miR-125b and clinical outcome, using the median as a cut-off value. The median follow-up time was 12.5 months (range 1-26). High levels of circulating miR-125b in patients with $\mathrm{MM}$ were associated with shorter EFS ( $\mathrm{P}=0.02$, Fig. 1D).

Effects of the expression of miR-125b on cellular growth, migration, invasion and apoptosis. The CCK-8 assay results showed that the viability of U266 and RPMI-8226 cells was inhibited by transfection of the miR-125b inhibitor (Fig. 2A). Cell growth in the miR-125b mimic group was significantly increased compared with that in the blank and NC groups $(\mathrm{P}<0.05)$. Furthermore, the present study examined whether treatment with the miR-125b inhibitor inhibited MM cell migration using a Transwell assay. Consistent with the CCK-8 assay, the Transwell assay demonstrated that the migration rate of cells transfected with miR-125b inhibitor 
A

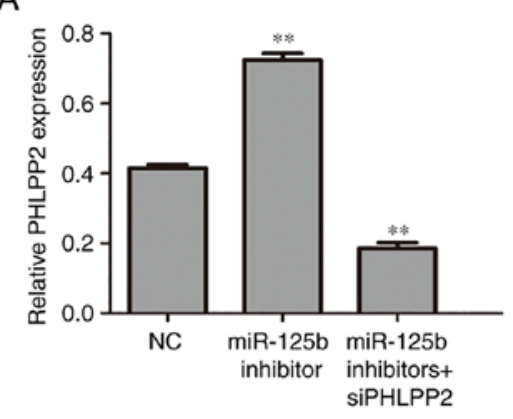

B $\rightarrow$ NC

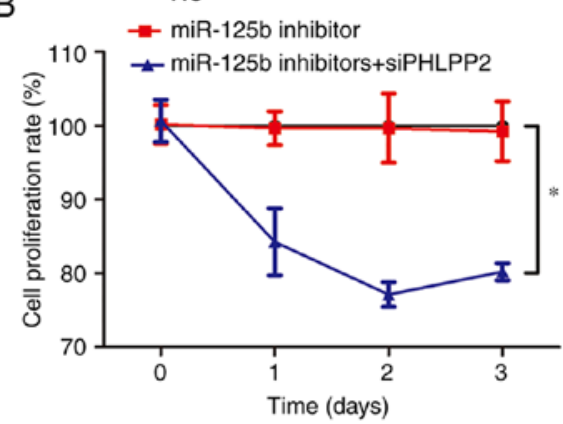

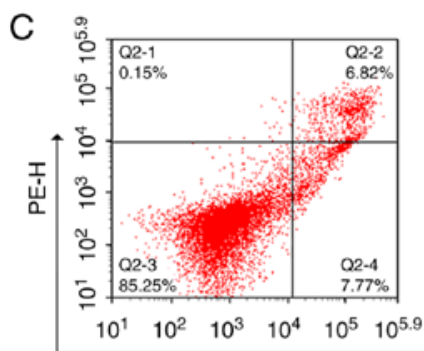

NC

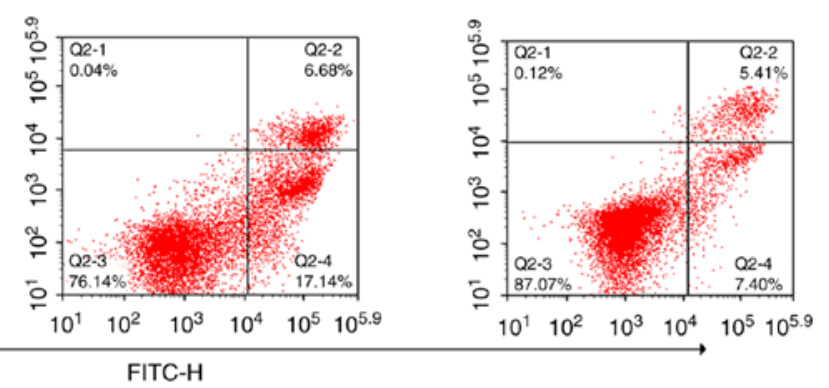

miR-125b inhibitor

miR-125b inhibitors+siPHLPP2

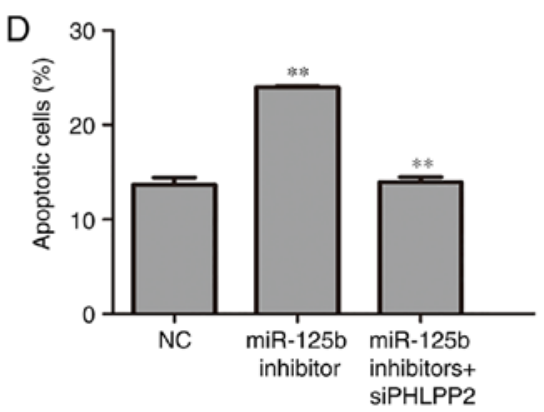

Figure 4. PHLPP2 reverses the effect of miR-125b. (A) mRNA levels of PHLPP2 were detected by reverse transcription-quantitative polymerase chain reaction analysis in U266 cells transfected with negative control, miR-125b inhibitor or miR-125b inhibitor + PHLPP2 siRNA. (B) Cell viability was assessed following transfection of PHLPP2 siRNA into miR-125b inhibitor U266 cells. (C and D) Apoptosis was analyzed via flow cytometry following PHLPP2 siRNA transfection into miR-125b inhibitor U266 cells. " $\mathrm{P}<0.01,{ }^{*} \mathrm{P}<0.05$. miR, microRNA; MM, multiple myeloma; PHLPP2, PH domain and leucine rich repeat protein phosphatase 2; siRNA, small interfering RNA; NC, negative control.

was inhibited (Fig. 2B). The number of migrated cells in the miR-125b inhibitor group was significantly lower than the numbers in the blank and $\mathrm{NC}$ groups $(\mathrm{P}<0.05)$. The cell invasion results showed that the number of invaded cells in the miR-125b inhibitor group was significantly lower than the numbers in the blank and NC groups (Fig. $2 \mathrm{C})(\mathrm{P}<0.05)$. In addition, the apoptotic rates in the miR-125b inhibitor group were $23.8 \%$ in the U266 cells and $19.6 \%$ in the RPMI-8226 cells (Fig. 2D). Compared with the two control groups, the apoptotic rate in the miR-125b inhibitor group was significantly higher $(\mathrm{P}<0.05)$ (Fig. 2E).

PHLPP2 as a target gene of miR-125b. On further analyzing the regulatory mechanism through which miR-125b promotes MM cell proliferation, the present study found that the tumor suppressor PHLPP2 was the target of miR-125b, as predicted by TargetScan (http://genes.mit.edu/targetscan.test/ucsc. html) (Fig. 3A). To determine whether miR-125b binds to the 3'-UTR of PHLPP2, a luciferase reporter assay was performed. As expected, the overexpression of miR-125b inhibited luciferase activity. By contrast, cells with a mutant PHLPP2 3'-UTR exhibited higher luciferase activity (Fig. 3B). To determine whether miR-125b downregulates PHLPP2 at the mRNA level, the expression of PHLPP2 was examined by RT-qPCR analysis. As shown in Fig. 3C and D, the mRNA expression of PHLPP2 was upregulated in MM cells transfected with miR-125b inhibitor. By contrast, the mRNA level of PHLPP2 was decreased in MM cells transfected with miR-125b mimic.

Protein expression of PHLPP2, AKT and p-AKT. In the U266 cells, the western blot analysis results showed that the expression of p-Akt in the miR-125b mimic group was downregulated compared with that in the blank and $\mathrm{NC}$ groups $(\mathrm{P}<0.05)$. miR-125b knockdown promoted the expression of PHLPP2, suggesting that miR-125b inhibited PHLPP2 (Fig. 3E and F). No statistically significant differences in protein expression were found between the blank and NC groups.

PHLPP2 reverses the effect of miR- $125 b$. To furthere whether PHLPP2 is a functional target of miR-125b, rescue experiments were performed by inhibiting PHLPP2. The U266 cells were cotransfected with miR-125b inhibitor and siPHLPP2. 

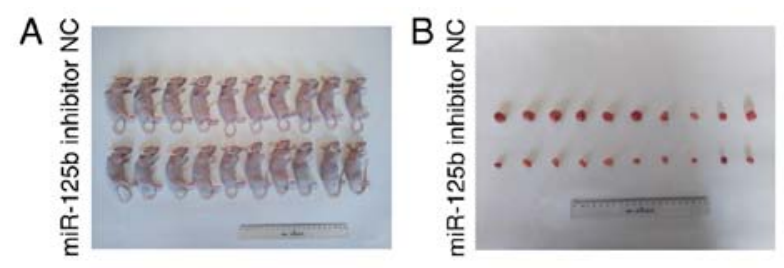

D
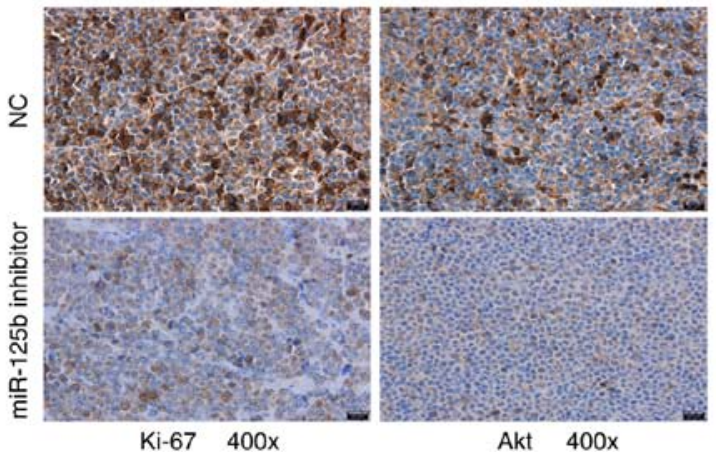

$\mathrm{F}$
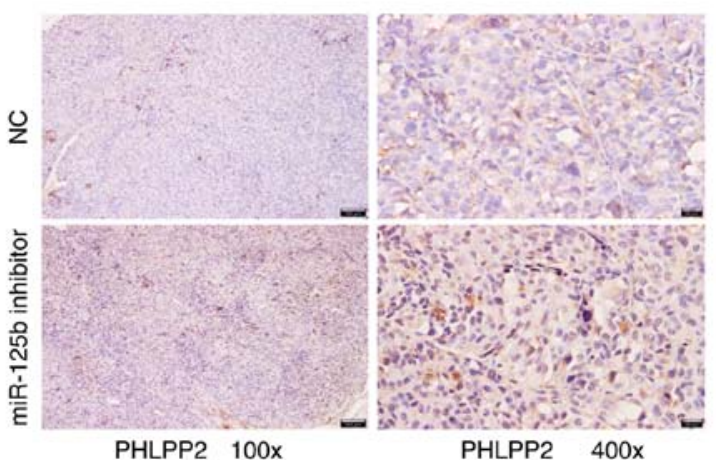
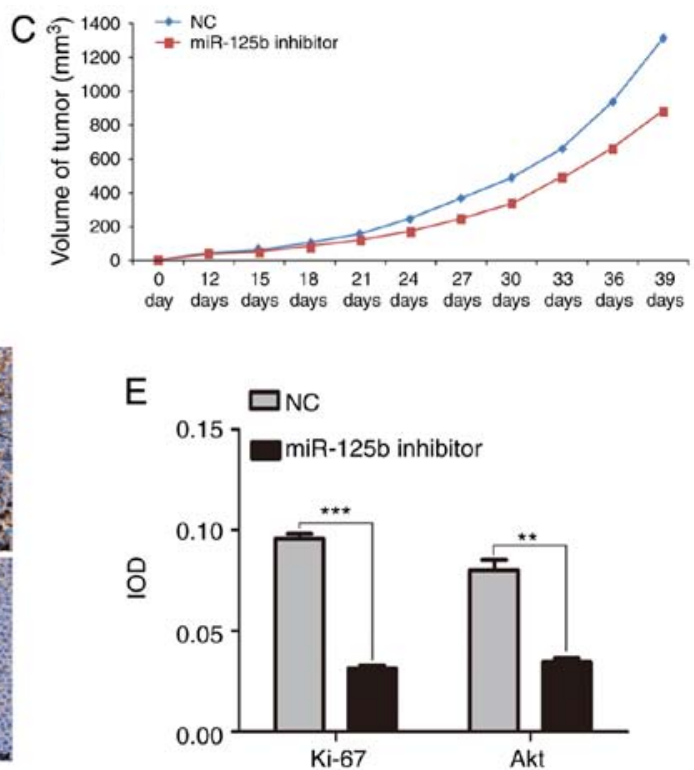

G

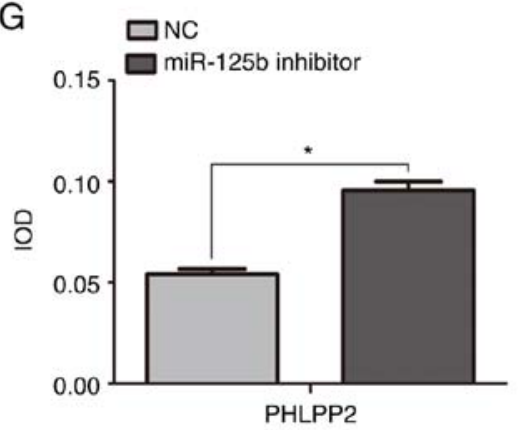

Figure 5. Effect of the expression of miR-125b on tumor growth in the nude mouse model. (A) Mice treated with miR-125b inhibitor or NC. (B) Tumors from mice treated with miR-125b inhibitor or NC. (C) Tumor volumes of mice treated with miR-125b inhibitor or NC (P<0.05). (D) Immunohistochemistry results showed that Ki-67 and Akt were mainly expressed in the NC. (E) Expression levels of Ki-67 and Akt were quantified as IOD values. (F) Images of immunohistochemical analysis of PHLPP2 in tumors from nude mice. $(\mathrm{G})$ Expression levels of $\mathrm{PHLPP} 2$ were quantified as IOD values. ${ }^{* * * *} \mathrm{P}<0.001,{ }^{* *} \mathrm{P}<0.01,{ }^{*} \mathrm{P}<0.05$. miR, microRNA; PHLPP2, PH domain and leucine rich repeat protein phosphatase 2; NC, negative control; IOD, integrated optical density.

Transfection with siPHLPP2 successfully decreased the expression of PHLPP2 in the cotransfected U266 cells (Fig. 4A, $\mathrm{P}<0.01)$. The CCK-8 assay indicated that the cell growth inhibition induced by miR-125b knockdown was relieved by transfection with siPHLPP2 (Fig. 4B, P<0.05). The results of the flow cytometry showed that cell apoptosis was increased in miR-125b inhibitor cells, whereas siPHLPP2 partially reversed this promotion in cell apoptosis (Fig. $4 \mathrm{C}$ and $\mathrm{D}, \mathrm{P}<0.05$ ).

Effect of the expression of miR-125b on tumor growth in a nude mouse model. The present study investigated whether the miR-125b inhibitor was able to inhibit tumor growth in the xenograft tumor model. Tumor growth was monitored for 39 days. The results showed that the tumor weights and volumes in the mice treated with miR-125b inhibitor were significantly reduced compared with those treated with $\mathrm{NC}(\mathrm{P}<0.05$; Fig. 5A-C). In addition, the immunohistochemistry results (Fig. 5D-G) showed that Ki-67 and Akt were mainly expressed in the NC group. The tumors from the miR-125b inhibitor group exhibited higher levels of PHLPP2 than the tumors from the NC group. The immunohistochemistry showed that treatment with miR-125b inhibitor reduced the expression of $\mathrm{Ki}-67$ and $\mathrm{Akt}$, and increased the expression of PHLPP2.

\section{Discussion}

$\mathrm{MM}$ is a $\mathrm{PC}$ disorder that represents a relatively high proportion of hematological malignancies. Despite significant progress in elucidating the mechanism of MM and identifying optimal treatments, the disease remains incurable, necessitating the development of novel and effective therapies (26). The therapeutic modulation of miRNAs that function as an oncogene or tumor suppressor may be a potential strategy for cancer treatment. The aim of the present study was to examine the roles of miR-125b in the proliferation, migration and invasion of MM cells.

The dysregulation of miRNA expression is important in cancer development, and circulating miRNAs serve as biomarkers for several malignancies (27-29). Aberrant miRNA expression profiles in plasma have also been reported in MM $(30,31)$. In the present study, miRNA microarray was used to compare the miRNA expression patterns of plasma samples from six patients with MM and six healthy controls. It 
was found that miR-125b, miR-4326, miR-4498, miR-490-3p and miR-7155-5p were overexpressed in the MM patient samples. Among these aberrant miRNAs, miR-125b had the highest degree of overexpression in samples from patients with MM compared with those from healthy control patients. Additionally, the expression of miR-125b was examined in MM cell lines and nPCs. miR-125b was upregulated in the MM cell lines compared with the nPCs.

Notably, miR-125b belongs to the miR-17-92 cluster and has been reported to act as an oncogene in several human malignancies. Wang et al demonstrated that miR-125b was overexpressed in nasopharyngeal carcinoma (32). Shen et al reported that the expression of miR-125b was markedly increased in type II diabetes mellitus (33). In addition, it has been shown that high levels of miR-125b are associated with shortened progression-free survival (PFS) times (34). In the present study, the expression of miR-125b in plasma was significantly higher in patients with stage III MM than in patients with stage I/II MM, suggesting the essential role of miR-125b in survival diagnosis. MM cell lines were utilized for subsequent loss-of-function experiments. The results showed a marked reduction in MM cell viability following transfection with an miR-125b inhibitor. The transfected cells also exhibited reduced migratory ability and an upregulated apoptosis level. Furthermore, the xenograft mouse model demonstrated the suppressive effects of miR-125b knockdown on tumor growth in vivo. The immunohistochemistry results showed that treatment with miR-125b inhibitor reduced the expression of Ki-67. The expression of $\mathrm{Ki} 67$ is associated with tumor cell proliferation and growth, and is widely used in routine pathological investigation as a proliferation marker. It has been reported that the inhibition of Ki67 either by antibodies or antisense oligonucleotides leads to the arrest of cell proliferation. Notably, antisense oligonucleotides and antibodies against p-Ki67 have been shown to inhibit progression of the cell cycle (35). Therefore, the suppression of Ki67 s may be a promising strategy in MM therapy. These data suggest that miR-125b is relevant to the development and progression of MM.

As an oncogenic pathway, the Akt pathway is frequently activated during tumorigenesis and is crucial in the growth, proliferation, migration and invasion of malignant cells $(36,37)$, making this pathway an attractive candidate for drug development (38). Studies have shown that PI3K/Akt pathway activation reduces levels and of p21Cip1 and increased the expression of cyclin D1 $(39,40)$, which regulate cell cycle progression through the G1 phase. In addition, activated phosphoinositide 3-kinase (PI3K) catalyzes 3,4-phosphatidylinositol trisphosphate phosphorylation and subsequently activates protein kinase Akt to promote cell growth and proliferation (36). Chen et al reported that the activation of AKT was associated with intrahepatic metastasis, tumor grade and a high proliferation index. Furthermore, the Akt pathway is a diagnostic and prognostic indicator of hepatocellular carcinoma invasion and metastasis, and a therapeutic target for this type of cancer (41). PHLPP2, a characterized member of the PHLPP family and suppressor of AKT signaling, exhibits an inhibitory effect on cell cycle progression and apoptosis in various tumor cell types (42).
PHLPP2 has been predicted to be a direct target of miR-125b; therefore, the present study confirmed this prediction via RT-qPCR, western blot and dual-luciferase reporter assays. The mRNA and protein levels of PHLPP2 were increased upon the downregulation of miR-125b in U266 cells. To confirm whether PHLPP2 is a target that mediates the function of miR-125b, a loss-of-function approach was used to functionally characterize the roles of PHLPP2 in the growth and apoptosis of MM. It was found that PHLPP2 silencing partially attenuated the effects of miR-125b inhibition on cell proliferation and apoptosis. Evidence suggests that the activation of PI3K/Akt by miR-181 is mainly mediated by the suppression of PHLPP2 (43). The results of the present study revealed that $\mathrm{p}-\mathrm{AKT}$ was downregulated in MM cells in which miR-125b was suppressed. A previous study examined the mechanism of miR-125b in the occurrence and progression of MM (44). The present study investigated the effect of miR-125b on the migration and apoptosis of MM, and on the Akt signal pathway. The prometastatic effect of miR-125b in MM was also examined, and a novel target gene of miR-125b was reported. The results demonstrated a novel role for miR-125b in AKT pathway activation by the direct suppression of PHLPP2.

In conclusion, the present study demonstrated that miR-125b regulated MM cell proliferation and migration by modulating the Akt signaling pathway. Therefore, this pathway may serve as a novel therapeutic target for MM. Notably, miR-125b suppressed the expression of PHLPP2, leading to activation of the AKT pathway. Elucidating the association between miR-125b and the Akt signaling pathway may assist in identifying novel molecular markers and potential therapeutic targets for MM treatment. The effects of miR-125b on MM cell proliferation, migration and invasion require confirmation using animal models in further investigations.

\section{Acknowledgements}

Not applicable.

\section{Funding}

This study was supported by the International Collaboration Fund of the National Science and Technology Committee of China (grant no. 2011DFA32820), the National Natural Science Fund Project (grant no. 81460037) and the Innovation Fund Project in Jiangxi Province (grant no. YC2016-B018).

\section{Availability of data and materials}

The datasets used during in the present study are available from the corresponding author upon reasonable request.

\section{Authors' contributions}

YJ performed the molecular biology experiments and drafted the manuscript. JD collected, analyzed and interpreted the data. JL performed the molecular biology experiments and revised the manuscript. GC conceived and designed the study. All authors read and approved the manuscript and agree to be 
accountable for all aspects of the research in ensuring that the accuracy or integrity of any part of the work are appropriately investigated and resolved.

\section{Ethics approval and consent to participate}

The study was approved by the Medical Research Ethics Committee of The First Affiliated Hospital of Nanchang University and written informed consent was obtained from all study subjects. The IRB number of human sample study approved by institutional committee is 2015(096). The use of mice in animal experiments was approved by the Affiliated Hospital of Nanchang University. The number of animal experiment approved by institutional committee is 2017(095).

\section{Patient consent for publication}

Consent to publish has been obtained from the participants.

\section{Competing interests}

The authors declare that they have no competing interests.

\section{References}

1. Sonneveld P, Avet-Loiseau H, Lonial S, Usmani S, Siegel D, Anderson KC, Chng WJ, Moreau P, Attal M, Kyle RA, et al: Treatment of multiple myeloma with high-risk cytogenetics: a consensus of the International Myeloma Working Group. Blood 127: 2955-2962, 2016.

2. Abdi J, Chen G and Chang H: Erratum: Drug resistance in multiple myeloma: Latest findings and new concepts on molecular mechanisms. Oncotarget 6: 7364, 2015.

3. Mimura N, Hideshima T and Anderson KC: Novel therapeutic strategies for multiple myeloma. Exp Hematol 43: 732-741, 2015.

4. Costa LJ, Brill IK, Omel J, Godby K, Kumar SK and Brown EE: Recent trends in multiple myeloma incidence and survival by age, race, and ethnicity in the United States. Blood Adv 1: 282-287, 2017.

5. Lionetti M, Biasiolo M, Agnelli L, Todoerti K, Mosca L, Fabris S, Sales G, Deliliers GL, Bicciato S, Lombardi L, et al: Identification of microRNA expression patterns and definition of a microRNA/mRNA regulatory network in distinct molecular groups of multiple myeloma. Blood 114: e20-e26, 2009.

6. Calin GA and Croce CM: MicroRNAs and chromosomal abnormalities in cancer cells. Oncogene 25: 6202-6210, 2006.

7. Calin GA and Croce CM: MicroRNA-cancer connection: The beginning of a new tale. Cancer Res 66: 7390-7394, 2006.

8. Seckinger A, Meißner T, Moreaux J, Benes V, Hillengass J, Castoldi M, Zimmermann J, Ho AD, Jauch A, Goldschmidt $\mathrm{H}$, et al: miRNAs in multiple myeloma-a survival relevant complex regulator of gene expression. Oncotarget 6 : 39165-39183, 2015.

9. Leotta M, Biamonte L, Raimondi L, Ronchetti D, Di Martino MT, Botta C, Leone E, Pitari MR, Neri A, Giordano A, et al: A p53-dependent tumor suppressor network is induced by selective miR-125a-5p inhibition in multiple myeloma cells. J Cell Physiol 229: 2106-2116, 2014.

10. Chi J, Ballabio E, Chen XH, Kusec R, Taylor S, Hay D, Tramonti D, Saunders NJ, Littlewood T, Pezzella F, et al: MicroRNA expression in multiple myeloma is associated with genetic subtype, isotype and survival. Biol Direct 6: 23, 2011.

11. Luo X, Gu J, Zhu R, Feng M, Zhu X, Li Y and Fei J: Integrative analysis of differential miRNA and functional study of miR-21 by seed-targeting inhibition in multiple myeloma cells in response to berberine. BMC Syst Biol 8: 82, 2014.

12. Esquela-Kerscher A: The lin-4 microRNA: The ultimate micromanager. Cell Cycle 13: 1060-1061, 2014.

13. Iorio MV and Croce CM: microRNA involvement in human cancer. Carcinogenesis 33: 1126-1133, 2012.
14. Garzon R and Marcucci G: Potential of microRNAs for cancer diagnostics, prognostication and therapy. Curr Opin Oncol 24: 655-659, 2012.

15. Ma Q, Chen Z, Jia G, Lu X, Xie X, and Jin W: The histone demethylase PHF8 promotes prostate cancer cell growth by activating the oncomiR miR-125b. Onco Targets Ther 8: 1979-1988, 2015.

16. Yu X, Shi W, Zhang Y, Wang X, Sun S, Song Z, Liu M, Zeng Q, Cui $S$ and Qu X: CXCL12/CXCR4 axis induced miR-125b promotes invasion and confers 5-fluorouracil resistance through enhancing autophagy in colorectal cancer. Sci Rep 7: 42226, 2017.

17. Wong TN and Link DC: $m i R-125 b$ promotes leukemogenesis via VEGFA. Blood 129: 1409-1410, 2017.

18. Yang Q, Wang Y, Lu X, Zhao Z, Zhu L, Chen S, Wu Q, Chen C, and Wang Z: MiR-125b regulates epithelial-mesenchymal transition via targeting Sema4C in paclitaxel-resistant breast cancer cells. Oncotarget 6: 3268-3279, 2015.

19. Zhang Y and Huang S: Up-regulation of miR-125b reverses epithelial-mesenchymal transition in paclitaxel-resistant lung cancer cells. Biol Chem, 2015.

20. Luo M, Tan X, Mu L, Luo Y, Li R, Deng X, Chen N, Ren M, Li Y, Wang L, et al: MiRNA-21 mediates the antiangiogenic activity of metformin through targeting PTEN and SMAD7 expression and PI3K/AKT pathway. Sci Rep 7: 43427, 2017.

21. Liu MH, Yang L, Liu XJ, Nie ZY, and Luo JM: Targeted suppression of miRNA-21 inhibit K562 cells growth through PTEN-PI3K/AKT signaling pathway. Zhonghua Xue Ye Xue Za Zhi 37: 982-986, 2016 (In Chinese).

22. Yang $X$, Cheng $Y$, Li P, Tao J, Deng X, Zhang X, Gu M, Lu Q, and Yin C: A lentiviral sponge for miRNA-21 diminishes aerobic glycolysis in bladder cancer T24 cells via the PTEN/PI3K/AKT/mTOR axis. Tumour Biol 36: 383-391, 2015.

23. Wang F, Li L, Chen Z, Zhu M, and Gu Y: MicroRNA-214 acts as a potential oncogene in breast cancer by targeting the PTEN-PI3K/Akt signaling pathway. Int J Mol Med 37: 1421-1428, 2016.

24. Anderson KC: Progress and paradigms in multiple myeloma. Clin Cancer Res 22: 5419-5427, 2016.

25. Livak KJ and Schmittgen TD: Analysis of relative gene expression data using real-time quantitative PCR and the $2^{-\Delta \Delta C_{\mathrm{T}}}$ method. Methods 25: 402-408, 2001.

26. Bates SE: Multiple myeloma: Multiplying therapies. Clin Cancer Res 22: 5418, 2016.

27. Gasparri ML, Casorelli A, Bardhi E, Besharat AR, Savone D, Ruscito I, Farooqi AA, Papadia A, Mueller MD, Ferretti E, et al: Beyond circulating microRNA biomarkers: Urinary microRNAs in ovarian and breast cancer. Tumour Biol 39: 1010428317695525, 2017.

28. Zeng Z, Chen X, Zhu D, Luo Z, and Yang M: Low expression of circulating MicroRNA-34c is associated with poor prognosis in triple-negative breast cancer. Yonsei Med J 58: 697-702, 2017.

29. Jiang X, Wang W, Yang Y, Du L, Yang X, Wang L, Zheng G, Duan W, Wang R, Zhang X, et al: Identification of circulating microRNA signatures as potential noninvasive biomarkers for prediction and prognosis of lymph node metastasis in gastric cancer. Oncotarget 8: 65132-65142, 2017.

30. Qu X, Zhao M, Wu S, Yu W, Xu J, Xu J, Li J and Chen L: Circulating microRNA 483-5p as a novel biomarker for diagnosis survival prediction in multiple myeloma. Med Oncol 31: 219, 2014.

31. Huang JJ, Yu J, LiJ Y, Liu YT and Zhong RQ: Circulating microRNA expression is associated with genetic subtype and survival of multiple myeloma. Med Oncol 29: 2402-2408, 2012.

32. Li LN, Xiao T, Yi HM, Zheng Z, Qu JQ, Huang W, Ye X, Yi H, Lu SS, Li XH and Xiao ZQ: MiR-125b increases nasopharyngeal carcinoma radioresistance by targeting $\mathrm{A} 20 / \mathrm{NF}-\kappa \mathrm{B}$ signaling pathway. Mol Cancer Ther 16: 2094-2106, 2017.

33. Shen Y, Xu H, Pan X, Wu W, Wang H, Yan L, Zhang M, Liu X, Xia $S$ and Shao Q: miR-34a and miR-125b are upregulated in peripheral blood mononuclear cells from patients with type 2 diabetes mellitus. Exp Ther Med 14: 5589-5596, 2017.

34. Piatopoulou D, Avgeris M, Marmarinos A, Xagorari M, Baka M, Doganis D, Kossiva L, Scorilas A and Gourgiotis D: miR-125b predicts childhood acute lymphoblastic leukaemia poor response to BFM chemotherapy treatment. Br J Cancer 117: 801-812, 2017.

35. Li LT, Jiang G, Chen Q and Zheng JN: Ki67 is a promising molecular target in the diagnosis of cancer (review). Mol Med Rep 11: 1566-1572, 2015. 
36. Yang Z, Fang S, Di Y, Ying W, Tan Y and Gu W: Modulation of NF- $\kappa \mathrm{B} / \mathrm{miR}-21 / \mathrm{PTEN}$ pathway sensitizes non-small cell lung cancer to cisplatin. PLoS One 10: e0121547, 2015.

37. Zhang Y, Zheng L, Ding Y, Li Q, Wang R, Liu T, Sun Q, Yang H, Peng S, Wang W and Chen L: MiR-20a induces cell radioresistance by activating the PTEN/PI3K/Akt signaling pathway in hepatocellular carcinoma. Int J Radiat Oncol Biol Phys 92: 1132-1140, 2015.

38. Carnero A, Blanco-Aparicio C, Renner O, Link W and Leal JF: The PTEN/PI3K/AKT signalling pathway in cancer, therapeutic implications. Curr Cancer Drug Targets 8: 187-198, 2008.

39. Medema RH, Kops GJ, Bos JL and Burgering BM: AFX-like forkhead transcription factors mediate cell-cycle regulation by ras and PKB through p2 $7^{\text {kipl }}$. Nature 404: 782-787, 2000.

40. Roy SK, Srivastava RK and Shankar S: Inhibition of PI3K/AKT and MAPK/ERK pathways causes activation of FOXO transcription factor, leading to cell cycle arrest and apoptosis in pancreatic cancer. J Mol Signal 5: 10, 2010.
41. Chen JS, Wang Q, Fu XH, Huang XH, Chen XL, Cao LQ Chen LZ, Tan HX, Li W, Bi J and Zhang LJ: Involvement of $\mathrm{PI} 3 \mathrm{~K} / \mathrm{PTEN} / \mathrm{AKT} / \mathrm{mTOR}$ pathway in invasion and metastasis in hepatocellular carcinoma: Association with MMP-9. Hepatol Res 39: 177-186, 2009.

42. Wei XE, Zhang FY, Wang K, Zhang QX and Rong LQ: Assembly of the FKBP51-PHLPP2-AKT signaling complex in cerebral ischemia/reperfusion injury in rats. Brain Res 1566: 60-68, 2014.

43. Strotbek M, Schmid S, Sanchez-Gonzalez I, Boerries M, Busch $\mathrm{H}$ and Olayioye MA: miR-181 elevates Akt signaling by co-targeting PHLPP2 and INPP4B phosphatases in luminal breast cancer. Int J Cancer 140: 2310-2320, 2017.

44. Gao D, Xiao Z, Li HP, Han DH and Zhang YP: The mechanism study of miR-125b in occurrence and progression of multiple myeloma. Cancer Med 7: 134-145, 2018. 